Journal of Mathematics and Statistics 3 (1): 21-23, 2007

ISSN 1549-3644

(C) 2007 Science Publications

\title{
Generalized Cauchy's Models and Generalized Integrals
}

\author{
Sabra Ramadan \\ Department of Mathematics, Jazan Teachers College, Ministry of Higher Education \\ The Kingdom of Saudi Arabia, Jazan- Abu Areesh, P.O. Box 203
}

\begin{abstract}
The space of generalized complex numbers $\mathrm{C}^{*}$ has been constructed. The Cachy's model in the space of new generalized functions is well defined. The generalized integral of new generalized function over the compact $K$ has been defined.
\end{abstract}

Key words: Cauchy's models, generalized complex numbers, generalized integral, ideal, algebra, topology

\section{INTRODUCTION}

Antonevich and Radyno ${ }^{[1]}$ gave the following general method of constructing algebras of new generalized functions:

Let $E$ - be some generalized function space and there is a some algebra $A$ of infinitely many differentiable functions such that $\mathrm{A} \subset \mathrm{E}$.

The multiplication of generalized functions $\eta, \mu \in \mathrm{E}$ will be defined by constructing a new algebra $\zeta$ and embedding (linear and injective mapping $\mathrm{j}: \mathrm{E} \rightarrow \zeta$, such that $j(u v)=j(u) j(v)$ for each $u, v \in A)$.

If we have the following objects:

1. $E$ - separated topological vector space;

2. Topological algebra $\mathrm{A} \subset \mathrm{E}$;

3. Some method of regularization define by a set of linear operators $\mathrm{R}_{\psi, \varepsilon}: \mathrm{E} \rightarrow \mathrm{A}, \quad \psi \in \phi, \varepsilon \in \varsigma$

(where $\phi$ - fixed set, $\varsigma$ - set with filter) so that $\forall \psi \in \phi, \mathrm{u} \in \mathrm{E}$

$\mathrm{R}_{\psi, \varepsilon}(\mathrm{u}) \rightarrow \mathrm{u}$ in since of topology of $E$.

Define $\mathrm{G}(\phi, \mathrm{A})=\{\mathrm{f}: \phi \mathrm{x} \varsigma \rightarrow \mathrm{A}\} \quad$ and $\mathrm{R}_{\mathrm{u}}$ the embedding of $E$ into $\mathrm{G}(\phi, \mathrm{A})$ :

$\mathrm{E}$ э $\mathrm{u} \rightarrow \mathrm{R}_{\mathrm{u}}(\phi, \mathrm{A}), \mathrm{R}_{\mathrm{u}}(\psi, \varepsilon) \equiv \mathrm{R}_{\psi, \varepsilon}(\mathrm{u})$

The elements $f_{1}, f_{2} \in G(\phi, A)$ are called weakly equivalent if $\forall \psi \in \phi, \mathrm{f}_{1}(\psi, \varepsilon)-\mathrm{f}_{2}(\psi, \varepsilon) \rightarrow 0$ in since of the topology of $E$.

In algebra $\mathrm{G}(\phi, \mathrm{A})$ define a sub algebra $\mathrm{G}^{*}(\phi, A)$ and some ideal $\mathrm{N}(\phi, \mathrm{A})$ and define the algebra $\zeta(\phi, \mathrm{A})=\mathrm{G}^{*}(\phi, \mathrm{A}) / \mathrm{N}(\phi, \mathrm{A})$.
Theorem 1: Let the sub algebra $G^{*}$ and the ideal $N$ satisfy the following conditions:

1. $\forall \mathrm{u} \in \mathrm{E}, \mathrm{R}_{\mathrm{u}} \in \mathrm{G}^{*}$;

2. The elements of $N$ are weakly equivalent of zero;

3. $\mathrm{R}_{\mathrm{u} . \mathrm{v}}-\mathrm{R}_{\mathrm{u}} \cdot \mathrm{R}_{\mathrm{v}} \in \mathrm{N}, \forall \mathrm{u}, \mathrm{v} \in \mathrm{A}$.

Then $E$ included in algebra $\zeta$ as a vector sub space and $A$ included in $\zeta$ as a sub algebra and if the operator of differentiation $D$ defined in $A$ so that $\mathrm{D}\left(\mathrm{G}^{*}\right) \subset \mathrm{G}^{*}$ and $\mathrm{D}(\mathrm{N}) \subset \mathrm{N}$ then the operator $D$ is well defined on $\zeta$ and $A$ embedded in $\zeta$ with the operator $D$.

Theorem 2: If there is an algebra $\zeta$ and embedding $\mathrm{j}: \mathrm{E} \rightarrow \zeta$, such that $A$ included in $\zeta$ as a sub algebra. Then for each $\mathrm{R}_{\psi, \varepsilon}, \psi \in \phi, \varepsilon \in \zeta$, there are a sub algebras $G^{*}$ and $N$ that satisfy the conditions of Theorem 1 and $\zeta=\mathrm{G}^{*} / \mathrm{N}$ isometric of the smallest sub algebra containing $E$.

Generalized complex numbers: Following the Antonevich -Radyno general method of constructing algebras of new generalized functions in $^{[2-7]}$ were constructed many algebras of new generalized functions as: $\zeta(\xi(\mathrm{R})), \zeta(\mathrm{D}(\mathrm{R})), \zeta(\mathrm{Z}(\mathrm{R})), \zeta(\mathrm{S}(\mathrm{R})), \zeta(\Pi(\mathrm{R}))$.

where the elements of the algebra $\zeta(\mathrm{M})$ are equivalence classes of sequences of elements in $M$.

To define the value of the element $\eta \in \zeta(\mathrm{M})$ at a some point $\mathrm{x}_{0}$ and to define and study some mathematical models, for example as Cachy's problem:

Corresponding Author: $\quad$ Department of Mathematics, Jazan Teachers College, Ministry of Higher Education, The Kingdom of Saudi Arabia, Jazan- Abu Areesh, P.O. Box 203 
$\left\{\begin{array}{l}\mathrm{Du}=\mathrm{au} \\ \mathrm{u}(0)=\mathrm{c}\end{array}\right.$

We define the generalized complex numbers correspondence to the space of new generalized functions $\zeta$ by the following way:

Let $G(C)$ - be the set of all sequences of complex numbers. Define $G^{*}(C)$ as the set of all sequences $\left(\mathrm{z}_{\mathrm{k}}\right) \in \mathrm{G}(\mathrm{C})$ that is there are a natural number $\mathrm{j} \in \mathrm{N}$ and a constant $\sigma_{1}>0$, such that $\left|\mathrm{z}_{\mathrm{k}}\right|<\sigma_{1} \mathrm{k}^{\mathrm{j}}$ for each $k$ in the domain of sequence $\left(\mathrm{z}_{\mathrm{k}}\right)$. Define the set $\mathrm{I}^{*}(\mathrm{C})$ as the set of all sequences $\left(z_{k}\right) \in G(C)$ that is for each natural number $i \in \mathrm{N}$, there is a constant $\sigma_{2}>0$, such that $\left|\mathrm{z}_{\mathrm{k}}\right|<\sigma_{2} \mathrm{k}^{-\mathrm{i}}$ for each $k$ in the domain of sequence $\left(z_{k}\right)$.

\section{Theorem 3}

1. Each of sets $G(C), G^{*}(C)$ is an algebra;

2. The set $I^{*}(C)$ be an ideal in the algebra $G^{*}(C)$.

Proof: We prove 2. Suppose that $\lambda=\left(\lambda_{\mathrm{k}}\right)$ be an elements in $I^{*}(\mathrm{C})$ it implies that is for each a natural number $i \in \mathrm{N}$, there is a constant $\sigma_{1}>0$, such that $\left|\lambda_{\mathrm{k}}\right|<\sigma_{1} \mathrm{k}^{-\mathrm{i}}$ for each $\mathrm{k}$ in the domain of sequence $\left(\lambda_{\mathrm{k}}\right)$ and let $\eta=\left(\eta_{k}\right) \in G^{*}(C)$ which implies that there are a natural number $\mathrm{j} \in \mathrm{N}$ and a constant $\sigma_{2}>0$, such that $\left|\eta_{\mathrm{k}}\right|<\sigma_{2} \mathrm{k}^{\mathrm{j}}$ for each $k$ in the domain of sequence $\left(\eta_{\mathrm{k}}\right)$. The inequality $\left|\eta_{\mathrm{k}} \lambda_{\mathrm{k}}\right| \leq\left|\eta_{\mathrm{k}}\right|\left|\lambda_{\mathrm{k}}\right| \leq \sigma_{2} \mathrm{k}^{\mathrm{j}} \sigma_{1} \mathrm{k}^{-\mathrm{i}}=\sigma_{2} \sigma_{1} \mathrm{k}^{\mathrm{j}-\mathrm{i}}$ implies that $\eta \lambda=\left(\eta_{\mathrm{k}} \lambda_{\mathrm{k}}\right) \in \mathrm{I}^{*}(\mathrm{C})$.

The proof of 1 is similar.

Define the algebra of generalized complex numbers as a factor spaces

$\mathrm{C}^{*}=\mathrm{G}^{*}(\mathrm{C}) / \mathrm{I}^{*}(\mathrm{C})$.

The following theorem shows the importance of the construction of the algebra $\mathrm{C}^{*}$ :

\section{Theorem 4}

1. If $\mathrm{h}=\left(\mathrm{h}_{\mathrm{k}}\right) \in \mathrm{G}^{*}(\xi(\mathrm{R}))$ and $\mu_{0} \in \mathrm{R}$, then $\mathrm{h}\left(\mu_{0}\right)=\left(\mathrm{h}_{\mathrm{k}}\left(\mu_{0}\right)\right) \in \mathrm{G}^{*}(\mathrm{C})$

2. If $\eta=\left(\eta_{\mathrm{k}}\right) \in \mathrm{I}^{*}(\xi(\mathrm{R}))$ and $\mu_{0} \in \mathrm{R}$ then $\eta\left(\mu_{0}\right)=\left(\eta_{\mathrm{k}}\left(\mu_{0}\right)\right) \in \mathrm{I}^{*}(\mathrm{C})$.

Proof: It is not difficult to prove this theorem by using the definitions of the space
$\zeta(\xi(\mathrm{R}))=\mathrm{G}^{*}(\xi(\mathrm{R})) / \mathrm{I}^{*}(\xi(\mathrm{R}))$ constructed $\mathrm{in}^{[2]}$ and by the definition of the algebra of generalized complex numbers $\mathrm{C}^{*}=\mathrm{G}^{*}(\mathrm{C}) / \mathrm{I}^{*}(\mathrm{C})$ defined above.

Now we can define the value of the new generalized function $h \in \zeta(\xi(R))$ at each point $\mu_{0} \in R$ as a generalized complex number $\mathrm{h}\left(\mu_{0}\right)=\left(\mathrm{h}_{\mathrm{k}}\left(\mu_{0}\right)\right) \in \mathrm{C}^{*}$, where $\left(\mathrm{h}_{\mathrm{k}}\right)$ be any representative of the new generalized function $\mathrm{h} \in \zeta(\xi(\mathrm{R}))$.

We define the embeddings of the set of all real numbers $R$ and the set of all complex numbers $C$ into the space of complex generalized numbers $\mathrm{C}^{*}=\mathrm{G}^{*}(\mathrm{C}) / \mathrm{I}^{*}(\mathrm{C})$ by the following way:

$\mathrm{k}_{1}: \mathrm{x} \in \mathrm{R} \rightarrow\left(\mathrm{x}_{\mathrm{k}}+0 \mathrm{i}\right) \in \mathrm{C}^{*}$, where $\mathrm{x}_{\mathrm{k}}=\mathrm{x} \forall \mathrm{k}$.

$k_{2}: z \in C \rightarrow\left(z_{k}\right) \in C^{*}$, where $z_{k}=z \forall k$.

The space $\zeta(\xi(\mathrm{R}))$ together with the space $\mathrm{C}^{*}$ we will denote by $\left(\zeta(\xi(\mathrm{R})), \mathrm{C}^{*}\right)$.

So the Cauchy's model in the space of new generalized functions is well defined in $\left(\zeta(\xi(\mathrm{R})), \mathrm{C}^{*}\right)$ and has a general form:

$\left\{\begin{array}{l}D u=v u \\ u(0)=z^{*} \\ u, v \in \zeta(\xi(R)), z^{*} \in C^{*}\end{array}\right.$

Moreover there arise many mathematical models in the space $\left(\zeta(\xi(R)), C^{*}\right)$ which have mathematical sense. For example the following models

$M_{n}=\left\{\begin{array}{l}D v=\delta^{n} v, \text { where } \delta-\text { theDirac function } \\ u(a)=b \\ v \in \zeta(\xi(R)), a, b \in C^{*}\end{array} n=2,3,4, \ldots .\right.$.

has a mathematical sense in the algebra $\left(\zeta(\xi(R)), C^{*}\right)$.

Generalized integrals: We define the integral in the space $\left(\zeta(\xi(R)), C^{*}\right)$ as the following:

Let $\mathrm{K} \subset \mathrm{R}$ be any compact set and $\eta \in \zeta(\xi(\mathrm{R}))$, define the integral of $\eta$ over the compact $K$ ( which we denote by $\left.\int_{\mathrm{K}}^{\vec{\eta}} \eta(\mathrm{x}) \mathrm{dx}\right)$ in the following way:

$\int_{\mathrm{K}}^{\overrightarrow{1}} \eta(\mathrm{x}) \mathrm{dx}=\left(\int_{\mathrm{K}} \eta_{\mathrm{k}}(\mathrm{x}) \mathrm{dx}\right)$,

where $\left(\eta_{k}\right)$-be any representative of $\eta$ 
Remark: The integral $\int_{\mathrm{K}} \eta(\mathrm{x}) \mathrm{dx}$ is well defined by virtue the following results:

\section{Theorem 5}

1. If $\left(\eta_{\mathrm{k}}\right) \in \mathrm{G}^{*}(\xi(\mathrm{R}))$, then $\left(\int_{\mathrm{K}} \eta_{\mathrm{k}}(\mathrm{x}) \mathrm{dx}\right) \in \mathrm{G}^{*}(\mathrm{C})$;

2. The integral $\int_{\mathrm{K}} \eta \mathrm{dx}$ is independent on $\mathrm{a}$ representative $\left(\eta_{\mathrm{k}}\right)$;

3. If $\left(\lambda_{\mathrm{k}}\right) \in \mathrm{I}^{*}(\xi(\mathrm{R}))$, then $\left(\int_{\mathrm{K}} \lambda_{\mathrm{k}}(\mathrm{x}) \mathrm{dx}\right) \in \mathrm{I}^{*}(\mathrm{C})$.

\section{Proof:}

1. Since $\left(\eta_{k}\right) \in G^{*}(\xi(R))$, then there are $i \in N, d>0$, such that $\sup _{\mathrm{x} \in \mathrm{K}}\left|\eta_{\mathrm{k}}(\mathrm{x})\right| \leq \mathrm{dk}^{\mathrm{i}}$ for each $k$ in the domain of $\left(\eta_{k}\right)$.

Consider $\left|\int_{\mathrm{K}} \eta_{\mathrm{k}}(\mathrm{x}) \mathrm{dx}\right| \leq \int_{\mathrm{K}}\left|\eta_{\mathrm{k}}(\mathrm{x})\right| \mathrm{dx} \leq \mathrm{dk} \int_{\mathrm{K}}^{\mathrm{i}} \mathrm{dx}$, that is $\left(\int_{\mathrm{K}} \eta_{\mathrm{k}}(\mathrm{x}) \mathrm{dx}\right) \in \mathrm{G}^{*}(\mathrm{C})$.

2. Let $\left(\lambda_{\mathrm{k}}-\lambda_{\mathrm{k}}^{\prime}\right) \in \mathrm{I}^{*}((\xi(\mathrm{R}))$, then $\forall \mathrm{i} \in \mathrm{N}, \exists \mathrm{d}>0: \sup _{\mathrm{x} \in \mathrm{K}}\left|\lambda_{\mathrm{k}}(\mathrm{x})-\lambda_{\mathrm{k}}^{\prime}(\mathrm{x})\right| \leq \mathrm{dk}^{-\mathrm{i}}, \forall \mathrm{k}$, consider $\left|\left(\int_{\mathrm{K}} \lambda_{\mathrm{k}}(\mathrm{x}) \mathrm{dx}-\int_{\mathrm{K}} \lambda_{\mathrm{k}}^{\prime}(\mathrm{x}) \mathrm{dx}\right)\right|=$
$\left|\left(\int_{\mathrm{K}}\left(\lambda_{\mathrm{k}}(\mathrm{x})-\lambda_{\mathrm{k}}^{\prime}(\mathrm{x})\right) \mathrm{dx}\right)\right| \leq \mathrm{dk}^{-\mathrm{i}} \int_{\mathrm{K}} \mathrm{dx} \forall \mathrm{k}$. Which means that $\left(\int_{\mathrm{K}} \eta_{\mathrm{k}}(\mathrm{x}) \mathrm{dx}-\int_{\mathrm{K}} \eta_{\mathrm{k}}^{\prime}(\mathrm{x}) \mathrm{dx}\right) \in \mathrm{I}^{*}(\mathrm{C})$.

3. The proof of 3 is similar.

Definition: The generalized complex number $z^{*}$ with representative $\left(\int_{\mathrm{K}} \lambda_{\mathrm{k}}(\mathrm{x}) \mathrm{dx}\right)$ is called the generalized integral of new generalized function $\lambda \in \zeta(\xi(\mathrm{R}))$ over the compact $K$, that is:
$\mathrm{z}^{*}=\int_{\mathrm{K}}^{\overrightarrow{1}} \lambda(\mathrm{x}) \mathrm{dx}=\left(\int_{\mathrm{K}} \lambda_{\mathrm{k}}(\mathrm{x}) \mathrm{dx}\right)$, where $\left(\lambda_{\mathrm{k}}(\mathrm{x})\right)$ be any representative of $\lambda$.

The generalized integral defined above preserve many properties of usual integral defined in $\xi(R)$, for example the following properties are preserved:

1. $\int_{\mathrm{K}}^{\overrightarrow{1}}[\lambda(\mathrm{x}) \pm \eta(\mathrm{x})] \mathrm{dx}=\int_{\mathrm{K}}^{\overrightarrow{1}} \lambda(\mathrm{x}) \mathrm{dx} \pm \int_{\mathrm{K}}^{\overrightarrow{1}} \eta(\mathrm{x}) \mathrm{dx}$

2. $\int_{\mathrm{K}}^{\vec{a}} \lambda(\mathrm{x}) \mathrm{dx}=\mathrm{a} \int_{\mathrm{K}}^{\overrightarrow{1}} \lambda(\mathrm{x}) \mathrm{dx}, \mathrm{a} \in \mathrm{C}^{*}$.

\section{REFERENCES}

1. Antonevish, A.B., 1991. Ya.V. Radyno, Dokl. Acad. Nauk USSR, 318: 267- 270; English Transl. in Soviet Math. Dokl., 43.

2. Radyno, Ya.V., N.P. Tkan and S. Ramadan, 1993. Russian Acad. Sci. Dokl., 327: 1. American Mathematical Society. English Transl. in Russian Acad.Sci. Dokl. Math., 46: 414-417.

3. Radyno, Ya.V., A.V. Romashevskii and S. Ramadan, 1993. Byelorussian Ac.Sci. Reports.

4. Tkan, N.P. and S. Ramadan, 1992. Weak solutions of differential equations in Algebra of new generalized functions. Abstract of the Conference of Belarus Mathematicians, Grodno, 112.

5. Ramadan, S. and N.P. Tkan, 1992. Convolution and Fourier transform in algebras of new generalized functions. Abstracts of the Interrepuplican Scientific and practical conference of Young Scientific Workers, Minsk, pp: 209.

6. Ramadan, S., 1998. The new generalized function spaces L(D(R)) and L(Z(R)). Dirasat, 25: 20-24.

7. Ramadan, S., 2006. One algebra of new generalized functions. J. Math. \& Stat., (In Press). 